\section{Temporal numerosity estimates for word lists*}

\author{
DOUGLAS H. LAWRENCE \\ Stanford University, Stanford, California 93405
}

The Ss estimated the number of items seen when sequences of words were presented tachistoscopically at presentation rates from 4 to 16 words/sec. The degree of underestimation increased with increasing rates of presentation, and the maximum average counting rate was between 6 and 7 words/sec. In the second phase of the study, they were asked to recall as many words as possible from short sequences of words presented at rapid rates, as well as to estimate the number of words seen but not available for recall. The total number of words seen, as judged from these two reports, agreed closely with the counting rates. The number of words correctly recalled decreased with increasing presentation rates.

In his extensive monograph, $\mathrm{C}$. T. White (1963) documents the long and continuing interest in the idea that perception is intermittent. It has been assumed that there are natural units of duration called psychological moments. Successive stimulus events, all occurring within the limits of a given moment, are perceived as simultaneous. Only those stimulus events occurring during different moments are perceived as successive.

Much of the experimental evidence of perceptual intermittency stems from the work on temporal numerosity (White, 1963). In these studies, it is usual to present $S$ with a series of stimulus events, such as a sequence of stroboscopic light flashes. The number of events in a series and the rate of presentation is varied over a considerable range. When Ss are asked to count or estimate the number of flashes perceived, they consistently underestimate. Furthermore, when these estimates are converted to the number of flashes counted per second, the counting rate tends to be 6.7 flashes/ $/ \mathrm{sec}$, regardless of the presentation rate. The implication is that Ss perceive only a limited proportion of the flashes presented or else that all flashes occurring during a psychological moment are perceived as a single unit.

Recently, a similar phenomenon was observed, serendipitously, while Ss viewed a series of words exposed tachistoscopically at a rapid presentation rate (Lawrence, 1971). Although they reported that each item perceived appeared clear and distinct, they were surprised to learn that their estimate of the number seen was only half the number actually presented.

The present study is designed to check this observation. Ss are asked to count or estimate the number of words seen in sequences of various lengths when these are

* This work was supported by Research Grant HD-00897 from the National Institutes of Health. exposed tachistoscopically at presentation rates from 4 to 16 words/sec. The point of interest is whether or not stimuli of this type, in which each item in the sequence is discriminably different from every other one, produce the same limit on counting rate as do sequences of light flashes in which all stimulus events are identical. In the second stage of the study, Ss are required to recall all the words they can from short sequences of words presented in the same way and at the same rates as in the counting phase. They also are required to estimate the number of words seen but which are unavailable for recall. From these two reports, a second estimate of the count rate is made. In addition, the recall data provide a rough index of the item positions within a sequence that are clearly perceived.

\section{APPARATUS}

The n-channel stroboscopic tachistoscope used in this study has been fully described previously (Lawrence \& Sasaki, 1970). In brief, S, with his head positioned by a chin rest, fixates a viewing aperture in a vertical panel placed at a exposed in this aperture during the brief flash of a strob lamp. (According to the manufacture's specifications, the duration of the flash is less than $50 \mathrm{msec}$, and its peak intensity, when used at the half-intensity setting as in this study, is $750,000 \mathrm{cp}$.) The light from this lamp first passes through the bond paper on which the word is typed and then through a During the off period of the lamp, the word is moved out of the aperture and a new one centered in it. It is possible to interpolate one or more light flashes between the exposures of successive words. The experimental room is dark except for the light from a $10 \mathrm{~W}$ bulb in a reading lamp on E's side of the apparatus. The $S$ is shielded from this by means of a black wooden tunnel that encloses the front of distance of 15 in. A stimulus word is neutral Polaroid filter (HN32 X .010 in.). the apparatus as well as his head.

\section{STIMUL AND METHOD}

A pool of 500 familiar words, each six letters in length, was compiled (Gates, 1937). These were entered into a PDP-8 computer, programmed so that lists of any length could be drawn at random. The only restriction was that no word should occur twice on the same list. Each list was then typed as a vertical column of words centered on a sheet of bond paper. All words were in lower case with the first letters of successive words vertically aligned.

\section{Counting Sets}

For the presentation rate of 4 words/sec, two light flashes were interpolated between each exposure of a stimulus word. This ensures a flash rate of 12 flashes/ $/ \mathrm{sec}$, a value above the point at which flicker effects are annoying. The maximum feasible list length that can be used under these circumstances is 15 words. Therefore, 31 lists were constructed with each list length from 1 through 15 words occurring once except for the leingths of $2,3,4$, and 5 items, which were represented by five lists each. The reason for this duplication of short list lengths is explained below. These lists were randomly ordered within a set.

For the presentation rate of 8 words/sec, one flash was interpolated between successive stimulus exposures. This ensures a flash rate of 16 flashes/sec and permits a maximum list length of 21 words. Forty-one lists were constructed for this condition with each list length from 1 through 21 represented once except for lengths of $2,3,4,5$, and 6 , which were represented by five lists each.

At the presentation rates of 12 and 16 words/sec, no interpolated flashes were used. Consequently, the flash rates are the same as the corresponding presentation rates. For each presentation rate, 51 lists were constructed with list lengths from 1 through 31 represented once except for lengths of $2,4,6,8$, and 10 , which were represented by five lists each.

\section{Memory Sets}

Prior to constructing the memory lists, four exploratory Ss were run on a different set of counting lists in order to obtain estimates of the number of words they saw for each list length at each presentation rate. On list lengths of 10 words or less, they also were asked for immediate recall of as many words as possible. Three tentative conclusions were drawn from the resulting data: (1) count and recall scores were perfect with only one word on a list at all presentation rates, (2) the maximum 


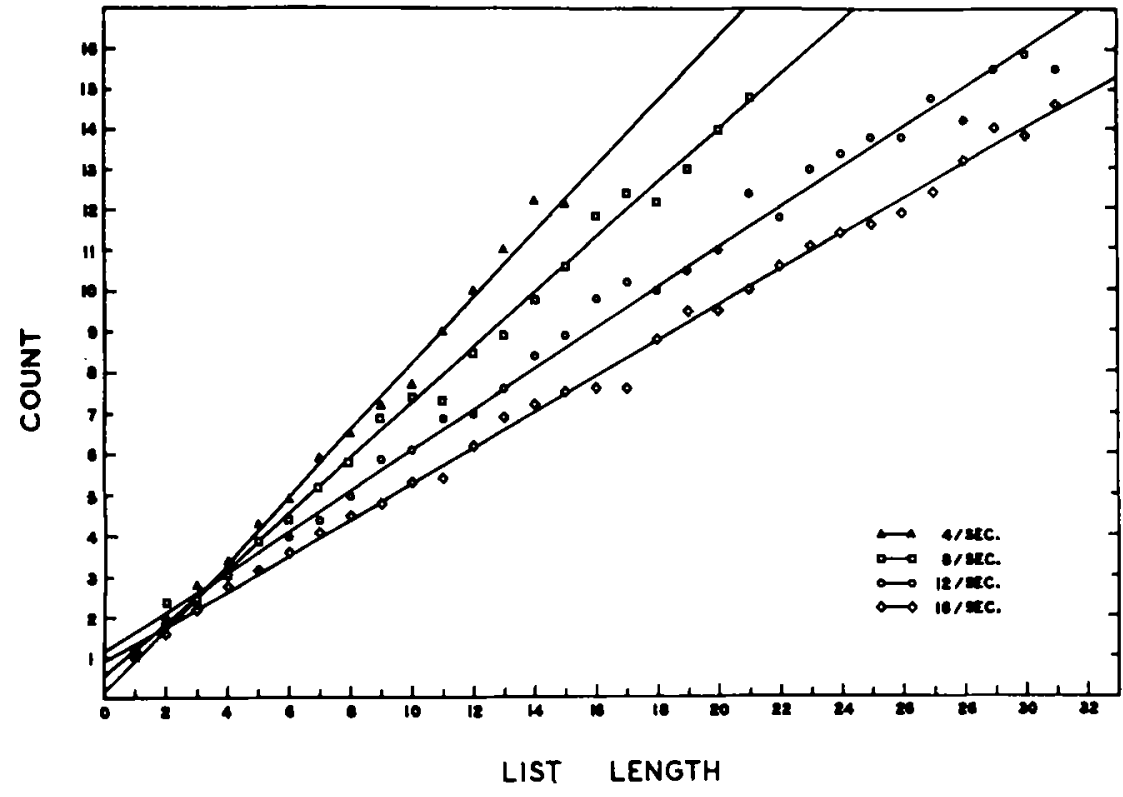

Fig. 1. Average number of items counted for word lists of various lengths.

number of words correctly recalled was five or six, even at the slow presentation rate of 4 words/sec, and (3) the counting score was quite accurate at 4 words/sec but underestimated the actual number by about half at 16 words/sec. On the basis of these results, it was decided to limit the memory list lengths to 2.5 words at 4 words/ sec, $2-6$ at 8 words $/ \mathrm{sec}$, and to the values of $2,4,6,8$, and 10 at 12 and 16 words/sec. Hopefully, Ss would never see more than five words, the limit of their immediate memory, on any of these lists. A set of memory lists was constructed for each presentation rate using the same pool of words as for the counting lists but with each of the above list lengths being represented 10 times in the appropriate set.

\section{Procedure}

At the beginning of each session, $S$ was permitted to dark adapt for $3 \mathrm{~min}$. He then initiated a trial, upon a signal from $\mathrm{E}$, by fixating the dimly lighted aperture and pressing the start switch. He first saw a preexposure field of 10 flashes, then the list of words, and finally a postexposure field of a variable number of flashes. If on any trial the words failed to appear in the center of the aperture, the trial data were discarded. This list was run again at the end of the set.

At the beginning of the first session, the nature of the task was explained, and $S$ was instructed in the counting proceedure. $\mathrm{He}$ was told that he should try to count each indication of a word even though he could not identify it. It was stressed that he should correct his report appropriately whenever he felt his counting rate was either leading or trailing the items actually seen. (But at no point in the experiment was $S$ informed with respect to the list lengths used or the accuracy of his report.) He was then given 10 practice trials on a special set of lists at the 4 words/sec presentation rate.

All Ss were given the four sets of counting lists in an increasing order of presentation rates. Two sets were given during each 50-min session. During the following two sessions, each $S$ counted the number of items on the memory lists, again in an increasing order of presentation rates. The $\mathrm{S}$ was informed that these lists were shorter on the average than the preceding ones, but he was not told the actual range of lists lengths used.

With repeated counting of short memory lists, it is possible that $S$ uses a different technique or strategy in estimating list length than he uses with the longer and more variable counting lists. It is for this reason that the short lists in the counting sets that were of the same length as those used in the memory sets occurred five times each. A comparison of the corresponding lists in the two sets should indicate if counting rate is influenced by the range of lengths used during a given session.

During the last four sessions of the study, $\mathbf{S}$ again was presented with the memory sets. He was instructed to give two different reports for each list. First, and most important, he was to recall immediately all the words he could. He was to recall them in any order he wished, and when he was uncertain of a word, he was to report any item that came to mind.
Second, he was to report the number of items he had seen but had completely forgotten during the process of recall or else the number of items that he had not seen clearly enough to identify. In scoring the recall data, $\mathrm{E}$ used a lenient criterion. Even though the word reported was not on the list, E scored it as correct if the sequence of letters in the reported item indicated which stimulus word had initiated it. If a recalled item did not meet this criterion, it was added to the second report. This double report procedure was designed to accomplish two things: (1) The correctly recalled words indicated the item positions within a list that were clearly perceived and (2) the sum of the number of items in the two reports (total score) provided an independent check on the counting scores for these lists. The four memory sets were given in an increasing order of presentation rates. As a reliability check, they were repeated in exactly the same order.

The Ss were eight paid graduate and undergraduate students, seven males and one female. All had normal or corrected $20 / 20$ vision.

\section{RESULTS}

For the counting sets, the scores were averaged across all Ss for each list length at each presentation rate. These values are shown in Fig. 1. Linear regression lines have been fitted to these averages by the method of least squares. It is apparent that the degree of underestimation of the actual list length increases directly with presentation rate. Even at 4 words/sec, Ss report only about $80 \%$ of the items exposed, and at 16 words/sec, they report less than half.

In analyzing the data, a linear regression line was fitted to the data for each $S$ at each presentation rate. Counting rates were obtained by multiplying each regression coefficient by the corresponding presentation rate. These counting rates were averaged across Ss. The average counting rates are $3.21,5.27,6.00$, and $6.76 \mathrm{items} / \mathrm{sec}$ for presentation rates of 4 , 8,12 , and 16 words/sec. All eight Ss have higher counting rates at 16 than at 12 words/sec, and six of the eight have higher values at 12 than at 8 words $/ \mathrm{sec}$. Thus, there is a tendency for counting rate to be correlated with presentation rate. It is doubtful that this is due to the partial confounding of flash rate and presentation rate in this study, in that both the 8- and 16-words/sec presentation rate used the same flash rate. Despite the increase in counting rate, the average values are in good agreement with those reported when the stimulus items are flashes of light (White, 1963). 


\begin{tabular}{lcccc}
\hline & \multicolumn{4}{c}{ Presentation Rate } \\
\cline { 2 - 5 } & 4 & 8 & 12 & 16 \\
\hline Memory Lists & 3.14 & 5.03 & 6.27 & 7.98 \\
Short Counting Lists & 3.00 & 5.44 & 6.13 & 7.34 \\
All Counting Lists & 3.21 & 5.27 & 6.00 & 6.76 \\
\hline
\end{tabular}

The counting rates for short list lengths are shown in Table 1. The pairs of values shown were obtained by computing the linear regression coefficient for each $S$ at each presentation rate for the memory sets and then for the lists of comparable length in the counting sets. These were converted to counting rates and averaged across Ss. For the limited data available, sign tests fail to show any significant difference between the four pairs of counting rates. Similarly, when the counting rates based on the memory sets are compared to those based on all list lengths in the counting sets, only the comparison at the presentation rate of 16 words/sec approaches significance $(p<.05)$. The implication is that the restriction on the range of list lengths in the memory sets has little or no influence on the counting rate.

Two scores were computed for each trial during the recall phase of this experiment-the number of words correctly recalled and the total score. The values for both of these, averaged across Ss and tests for each list length and presentation rate, are shown in Table 2 (Rows 1 and 3). Also included in this table are the average counting scores on these memory lists (Row 2).

The main impression from these data is the close correspondence between the counting scores and the total scores for the range of list lengths investigated. While 8 of the 19 comparisons are significant at the
.05 level, as determined by sign tests, the only apparent pattern in these data is in the somewhat more accurate estimates of list length based on the total score for lists containing six or fewer words. For longer lists, the counting score appears to be somewhat more accurate.

Because of the lenient criterion used in scoring the correctness of the recall data, the recall values given in Table 2 are somewhat inflated. Despite this, the average number of words recalled never excedes five; in fact, no $\mathrm{S}$ recalled more than six words from any list. The average number recalled increases directly with list length at all presentation rates. But for lists of a given length, the average recall score decreases with increasing presentation rate (cf. Aaronson, 1967). The one exception to this is for lists of two-item length, where recall is almost perfect at all presentation rates. This effect has been noted, also, when the stimulus events are light flashes (White, 1963).

Table 3 presents the serial position effects in the recall data for lists of various lengths at the presentation rate of 16 words/sec. (The same pattern of results occurs at 12 words $/ \mathrm{sec}$.) These percentages were computed by pooling the data from the two recall tests for lists of a given length. For each $S$, the number of times he recalled a word in a given position was counted, and this score was divided by the number of lists of that length that had

Table 2

Number of Words Correctly Recalled and Two Estimates of List Length for Memory Sets

\begin{tabular}{|c|c|c|c|c|c|c|}
\hline \multirow{2}{*}{$\begin{array}{l}\text { Presenta- } \\
\text { tion Rate } \\
\end{array}$} & & \multicolumn{5}{|c|}{ List Length } \\
\hline & & 2 & 3 & 4 & 5 & 6 \\
\hline $4 / \mathrm{Sec}$ & $\begin{array}{l}\text { Recall } \\
\text { Counting } \\
\text { Total }\end{array}$ & $\begin{array}{l}1.99 \\
2.08 \\
2.03\end{array}$ & $\begin{array}{l}2.97 \\
2.99 \\
3.04\end{array}$ & $\begin{array}{l}3.89 \\
3.73 \\
4.01\end{array}$ & $\begin{array}{l}4.45 \\
4.41 \\
4.83^{*}\end{array}$ & $\begin{array}{l}-- \\
-- \\
--\end{array}$ \\
\hline \multirow[t]{2}{*}{$8 / \mathrm{Sec}$} & $\begin{array}{l}\text { Recall } \\
\text { Counting } \\
\text { Total }\end{array}$ & $\begin{array}{l}1.98 \\
2.08 \\
2.03\end{array}$ & $\begin{array}{l}2.88 \\
2.88 \\
3.00\end{array}$ & $\begin{array}{l}3.44 \\
3.38 \\
3.69 *\end{array}$ & $\begin{array}{l}3.81 \\
4.10 \\
4.31^{*}\end{array}$ & $\begin{array}{l}4.09 \\
4.53 \\
4.72\end{array}$ \\
\hline & & \multicolumn{5}{|c|}{$\begin{array}{c}\text { List Length } \\
6\end{array}$} \\
\hline $12 / \mathrm{Sec}$ & $\begin{array}{l}\text { Recall } \\
\text { Counting } \\
\text { Total }\end{array}$ & $\begin{array}{l}1.99 \\
1.88 \\
1.99\end{array}$ & $\begin{array}{l}3.20 \\
2.94 \\
3.36^{*}\end{array}$ & $\begin{array}{l}3.72 \\
4.05 \\
4.31^{*}\end{array}$ & $\begin{array}{l}3.79 \\
5.05 \\
4.88\end{array}$ & $\begin{array}{l}4.20 \\
6.24 \\
5.31 *\end{array}$ \\
\hline $16 / \mathrm{Sec}$ & $\begin{array}{l}\text { Recall } \\
\text { Counting } \\
\text { Total } \\
\end{array}$ & $\begin{array}{l}1.94 \\
1.75 \\
1.96 \\
\end{array}$ & $\begin{array}{l}3.00 \\
3.13 \\
3.25\end{array}$ & $\begin{array}{l}3.41 \\
3.94 \\
4.09\end{array}$ & $\begin{array}{l}3.61 \\
4.91 \\
4.62^{*} \\
\end{array}$ & $\begin{array}{l}3.64 \\
5.85 \\
5.05^{*}\end{array}$ \\
\hline
\end{tabular}

*The total score and the corresponding counting score are significantly different $(p<.05)$, according to a sign test.
Serial Position Effects for the Recall Data at a Presentation Rate of 16 Words/Sec

\begin{tabular}{cccccc}
\hline $\begin{array}{c}\text { Serial } \\
\text { Position } \\
\text { of Item } \\
\text { in List }\end{array}$ & \multicolumn{4}{c}{$\begin{array}{c}\text { Average Percentage of } \\
\text { Correct }\end{array}$} \\
\hline 1 & 95.6 & 81.9 & 80.0 & 85.6 & 83.1 \\
2 & 98.1 & 75.0 & 73.8 & 81.9 & 58.8 \\
3 & & 48.8 & 49.4 & 38.8 & 33.8 \\
4 & & 94.4 & 17.5 & 15.6 & 24.4 \\
5 & & & 28.1 & 22.5 & 11.9 \\
6 & & & 87.5 & 11.9 & 17.5 \\
7 & & & & 18.8 & 26.2 \\
8 & & & & 88.1 & 6.9 \\
9 & & & & & 18.8 \\
10 & & & & & 76.9 \\
\hline
\end{tabular}

been given. These proportions were averaged across Ss and the result expressed as a percentage.

The most obvious trend in these data is the strong primacy-recency effect, with most of the recalled words coming from the first, second, and last item positions. The implication is that the words $S$ reports as having perceived, but which are unavailable for recall, come from the middle of the lists.

\section{DISCUSSION}

There is a close correspondence between the counting data in this study using word lists and the counting data in the studies summarized by White (1963), which used light flashes. The latter studies employed presentation rates between 10 and 30 flashes/sec and consistently obtained estimates of 6.7 flashes/sec. Despite the slight correlation between counting rate and presentation rate observed here, the maximum counting rate appears to be in the same range. It is true that for sequences of light flashes, the counting rate is considerably higher for sequences lasting less than $300 \mathrm{msec}$, an effect presumably due to adaptation level. It should be noted, however, that in the present study, $\mathrm{S}$ was exposed to a sequence of flashes of longer duration than this before he began to count the words. A further similarity between the two sets of results is the ability of $S$ to estimate accurately the number of events in a train of only two flashes or words over a wide range of presentation rates. In the present study, this is clearest in the estimates based on the total score of the recall tests.

Harter (1967), in his review of proposed neurological mechanisms that could account for intermittency in perception, has distinguished between two types of scanning models, one of which implies that all inputs during a given psychological moment are summated and a second that implies that only those inputs coincident with the scan are registered. If the concept of summation is taken to mean that two 
stimulus configurations presented successively, but on the same part of the retina, should be perceived as a single figure when both occur during a single moment, the present results would appear to be in opposition to the summation model. All Ss report that any item seen appears to be a sharp word-like configuration of letters. Exploratory studies indicate that the presentation rate must excede 20 words/sec before overprinting (i.e., the superposition of one set of letters on another) is seen. Consequently, if the counting rate is taken as an accurate index of the number of items perceived, one must assume that a large number of stimulus events have been suppressed at the higher presentation rates.

Unfortunately, the counting rate is an ambiguous indication of the number of items perceived. Landauer (1962) has shown that the maximum rate of silent counting in the absence of an external sequence of stimuli is approximately 6-7 numbers/sec. Thus, the limitation of the counting rate observed in this and other studies may be due to the slowness of the motor or output mechanisms rather than to limitations of the perceptual or input mechanisms. It was this consideration that led to the inclusion of the recall tests in the present study. The $S$ was set to identify and retain as many items as possible without counting. Then, from immediate memory and without time pressure, he reported a total score that provided an estimate of the number of items he had seen. It is true that this technique could be used only with short lists. Nonetheless, the close agreement between this estimate and the counting score suggests that the counting rate is a valid index of the number of events that $S$ processes per unit of time to the level of awareness. It is always possible, of course, that a more sensitive measure would indicate that immediate memory contains more events than can be measured by the double report procedure used here.

The pronounced primacy-recency effect in the recall data, plus the relatively small number of words correctly recalled at the higher presentation rates, makes it impossible to specify which item positions in the middle of a list are clearly perceived and which are missed. But the phenomenological reports of the Ss, that even those items they are aware of seeing but are unable to recall appear as distinct word-like configurations, suggest that any attempt to explain the observed counting rates in terms or backward- and forward-masking effects must allow for some form of temporal periodicity in the masking process. Some items in the middle of a list are clearly registered and others appear to have been missed entirely.

\section{REFERENCES}

AARONSON, D. Temporal factors in perception and short-term memory. Psychological Bulletin, 1967, 73, 130-144.

GATES, A. I. A list of spelling difficulties in 3876 words. New York: Teachers College, Columbia University, 1937.

HARTER, M. R. Excitability cycles and cortical scanning: A review of two hypotheses of central intermittency in perception. Psychological Bulletin, 1967, 68, 47-58.

LANDAUER, T. Rate of implicit speech. Perceptual \& Motor Skills, 1962, 15, 646.

LAWRENCE, D. H. Two studies of visual search for word targets with controlled rates of presentation. Perception \& Psychophysics, $1971,10,85-89$.

LAWRENCE, D. H., \& SASAKI, E. H. An n-channel tachistoscope for typewritten stimuli. Behavior Research Methods \& Instrumentation, 1970, 2, 63-65.

WHITE, C. T. Temporal numerosity and the psychological unit of duration. Psychological Monographs, 1963, 77(12, Whole No. 575).

(Accepted for publication October 18, 1970.) 